\title{
Modelagem e Estimação de Parâmetros Genéticos e Fenotípicos para Pesos do Nascimento à Seleção (378 dias) de Machos Nelore ${ }^{1,2}$
}

\author{
Joslaine Noely dos Santos Gonçalves Cyrillo ${ }^{3,4}$, Maurício Mello de Alencar ${ }^{4,5}$, Alexander \\ George Razook ${ }^{3}$, 4 , Maria Eugênia Zerlotti Mercadante ${ }^{3}$, Leopoldo Andrade de Figueiredo ${ }^{3}$
}

RESUMO - Parâmetros genéticos para pesos (17.942 observações), obtidos em intervalos de 60 dias, do nascimento ao momento da seleção (378 dias de idade), de 2.582 animais machos da raça Nelore, foram estimados em análises univariadas pelo método da máxima verossimilhança restrita. Os modelos de análise incluíram os efeitos fixos de grupo de contemporâneos, mês de nascimento, idade da mãe e idade na pesagem como covariável. Três modelos diferindo nos efeitos aleatórios foram testados: o modelo1 (M1) ajustou para os efeitos genéticos aditivos direto e materno e de ambiente permanente materno; no modelo 2 (M2), excluiu-se o efeito genético materno; e o modelo 3 (M3) ajustou somente para o efeito genético aditivo direto. O teste de razão de verossimilhança (LRT) detectou diferenças significativas para todas as idades, de M2 e M1 com o modelo simples (M3), evidenciando a importância dos efeitos maternos. Com exceção do peso ao nascer $(0,40)$, valores baixos $(0,05$ a 0,12$)$ de $\mathrm{h}^{2}$ foram estimados em M1 e M2 para os pesos até os 8 meses de idade. Após esse período a herdabilidade foi maior, chegando a 0,28 aos 13 meses de idade. As estimativas da fração da variância total, em decorrência do efeito de ambiente permanente materno, foram altas e permaneceram praticamente inalteradas nos modelos 1 e 2 . Efeitos maternos, não necessariamente decompostos (em genético aditivo e ambiente permanente), influenciaram o crescimento de animais machos da raça Nelore. Modelos nos quais foram incluídos efeitos maternos, além do genético aditivo direto, foram mais adequados para descrever a trajetória das variâncias ao longo das fases iniciais de crescimento de machos Nelore.

Palavras-chave: bovinos de corte, componentes de variância, efeitos maternos, herdabilidade, idades subseqüentes, medidas repetidas

\section{Modeling and Estimation of Genetic Parameters for Weights from Birth to Selection Age (378 Days) of Nellore Males}

\begin{abstract}
Genetic parameters for weights (17, 942 records), obtained in intervals of 60 days, from the birth to selection (378 days of age), of 2,582 males of the Nellore breed was estimated in univariate analyses by the Maximum Restricted Likelihood method. The models of analysis models included the fixed effects of contemporary groups, month of birth, mother age and age when the weights were collected as covariate. Three models differing in random effects were tested: the model 1 (M1) was adjusted for the direct and maternal addictive genetic effects and maternal permanent environment; in model 2 (M2) the maternal genetic effect was excluded; and the model 3 (M3) was only adjusted for the direct addictive genetic effect. The test of likelihood (LRT) detected significant differences, for all the ages, of M2 and M1 in relation to the simple model (M3), evidencing the importance of the maternal effects. Except for the birth weight (0.40), low values (0.05 to 0.12 ) of $\mathrm{h}^{2}$ were found for M1 and M2 until 8 months of age and, after this period, reasonable increase could be observed, reaching 0.28 until 13 months of age. The estimates of the total variance fraction, due to the effect of maternal permanent environment, were high and practically became unaffected between the models 1 and 2. Maternal effects, not necessarily decomposed (in genetic addictive and permanent environment), affected the Nellore males growth. Models that contemplate maternal effects, besides the genetic addictive direct effects, are more realistic to describe the trajectory of the variances in the initial phases of growth of Nelore male calves.
\end{abstract}

Key Words: beef cattle, heritability, maternal effects, repeated records, subsequent ages, variance components

\footnotetext{
1 Parte do projeto NRP-164.

2 Parte da tese apresentada ao Programa de Pós-Graduação em Produção Animal - UNESP - Jaboticabal, SP.

3 Pesquisador Científico - Instituto de Zootecnia - Sertãozinho, SP.

${ }^{4}$ Bolsista do CNPq.

5 Pesquisador da Embrapa Pecuária Sudeste - São Carlos, SP (mauricio@cppse.embrapa.br).
} 


\section{Introdução}

A decomposição dos componentes da variância fenotípica em variância genética e ambiental é baseada no princípio de que a semelhança fenotípica entre parentes fornece informações a respeito do grau de diferenciação genética entre eles. O componente aditivo da variância genética é de particular interesse, por ser o determinante primário do grau no qual a progênie se assemelha aos pais, sendo o que governa a taxa de resposta de um caráter à seleção (Lynch \& Walsh, 1997).

Em bovinos de corte, componentes de variância de características de crescimento são obtidos usualmente por meio de pesos padronizados em determinadas idades, como peso aos 210 dias (P210), peso aos 378 dias (P378), e outros. Esse tipo de avaliação tem se mostrado eficiente, uma vez que os avanços utilizando essa metodologia foram efetivos. Ganhos genéticos obtidos no Projeto de Melhoramento Genético das Raças Zebuínas de Sertãozinho (PMGRZS) foram em torno de 1,9 kg/ano para P210 (machos e fêmeas), 2,55 kg/ano para P378 (machos) e 2,99 kg/ano para P550 (fêmeas) no período de 1981 a 2000 (Razook, 2002). Entretanto, esse processo assume que o crescimento nesses intervalos é linear, e, para tanto, a amplitude das diferenças de idade dentro de cada idade padrão deve ser a mínima possível, exigindo, entre outros, que fatores de correção e/ou modelos de ajuste sejam adequados. Mesmo assim, provavelmente, ainda estar-se-ia incorrendo em algum erro, que seria assumir que, em toda a amplitude de idade considerada, as expressões dos efeitos genéticos e ambientais seguem o mesmo padrão, o que na realidade não ocorre (Van Der Werf \& Schaeffer, 1997). Análises considerando intervalos pequenos de idade, como 10, 20 ou 30 dias, poderiam ser viáveis e úteis não fosse o problema de obtenção de bancos de dados com número de registros e estruturas populacionais adequadas em cada uma dessas idades.

Metodologias recentes, como modelos de regressão aleatória (MRA) e funções de covariâncias (FC), são apresentadas atualmente como alternativas para análises de características obtidas repetidamente ao longo da vida do animal, também denominadas dados longitudinais (Kirkpatrick et al., 1990, 1994, 2002).

O PMGRZS acumulou, ao longo de 26 anos, um acervo formado por cerca de quatro gerações de seleção de bovinos de corte, delineado de modo que a composição das famílias possa oferecer estrutura adequada para a decomposição das fontes de variação genéticas e ambientais no decorrer do crescimento. Desde 1981 (primeira progênie), são coletadas sistematicamente informações de pesos em diferentes fases do crescimento dos bovinos, as quais, até o momento, não foram utilizadas na sua totalidade. Nesse contexto, pouco se conhece a respeito das variações genética e fenotípica existentes na trajetória completa, compreendida do nascimento à seleção de animais machos da raça Nelore.

Conduziu-se este trabalho com os objetivos de: 1) definir modelos genética e estatisticamente adequados que permitam descrever as estimativas dos parâmetros genéticos e fenotípicos do peso corporal em fases adjacentes do crescimento de machos da raça Nelore; 2) decompor adequadamente efeitos genéticos e ambientais e obter parâmetros que possam servir de comparação aos modelos de regressão aleatória; e 3) investigar e conhecer a trajetória dos componentes genéticos e ambientais que descrevem o crescimento do nascimento ao momento da seleção (378 dias de idade) de machos Nelore.

\section{Material e Métodos}

Os dados utilizados no presente estudo foram provenientes dos rebanhos da raça Nelore pertencentes ao PMGRZS da Estação Experimental de Zootecnia de Sertãozinho, unidade de pesquisa do Instituto de Zootecnia. O Centro está localizado ao norte do Estado de São Paulo, região que está $548 \mathrm{~m}$ do nível do mar e apresenta média anual de temperatura e precipitação de $23,61^{\circ} \mathrm{C}$ e $1312 \mathrm{~mm}$, respectivamente.

O processo de reestruturação do rebanho Nelore para dar início ao PMGRZS se deu em 1976, com a introdução de novas linhagens. O intuito desse procedimento foi aumentar a variabilidade genética e evitar, no futuro, níveis de endogamia indesejáveis. Em 1980, 350 fêmeas foram aleatoriamente divididas em três rebanhos denominados: Rebanho Controle $(\mathrm{NeC})$, formado por 60 matrizes, Rebanho Seleção (NeS), com 120 matrizes; e Rebanho Tradicional $(\mathrm{NeT})$, com 170 matrizes. Os touros fundadores foram os nascidos entre 1977 e 1980 e que alcançaram o diferencial de seleção máximo no peso padronizado aos 550 dias. No rebanho controle, foram utilizados animais que tiveram diferenciais de seleção nulos ou

R. Bras. Zootec., v.33, n.6, p.1405-1415, 2004 
próximos de zero, nesse mesmo caráter. Como as primeiras progênies dos rebanhos $\mathrm{NeC}, \mathrm{NeS}$ e $\mathrm{NeT}$ nasceram em 1981, dados de animais nascidos em anos anteriores foram identificados como rebanho original $(\mathrm{NeO})$.

O PMGRZS sofreu algumas alterações ao longo dos anos, mas as bases fundamentais de sua metodologia com relação à diminuição do intervalo de gerações e os critérios de seleção em machos e fêmeas, além da preocupação com a manutenção de baixos níveis de endogamia, permaneceram inalteradas (Razook et al., 1996).

A seleção é efetuada com base no maior diferencial de seleção para peso padronizado aos 378 dias (P378), obtido em prova de ganho de peso (PGP), dentro do grupo de contemporâneos. Os animais dos rebanhos $\mathrm{NeC}$, NeS e NeT permanecem sob os mesmos procedimentos de manejo durante a maior parte do ano, com exceção do período de estação de monta, quando são separados em lotes de acasalamento e mantidos em piquetes separados. A estação de monta ocorre de novembro a fevereiro e os nascimentos a partir da segunda quinzena de agosto até meados de novembro de cada ano. O desmame é feito em duas etapas: em abril, para animais nascidos nos dois primeiros meses da estação de nascimentos e em maio, para os nascidos no último mês, sendo que, em ambos os casos, os bezerros possuem, em média, sete meses (210 dias) de idade.

Após o desmame, os bezerros machos são encaminhados à PGP, onde permanecem por 168 dias segundo normas descritas por Razook et al. (1997). No início da PGP, são pesados em jejum alimentar e hídrico e seus pesos são padronizados para a idade de 210 dias. Os animais são pesados a cada 56 dias e o ganho de peso durante os últimos 112 dias é utilizado para obter o peso padronizado aos 378 dias (P378). Mais detalhes da fundação, execução e dos resultados do referido programa são descritos por Razook et al. (1993, 1998), Cyrillo et al. (2000) e Mercadante et al. (2003).

Neste estudo foram utilizados dados de animais machos nascidos de 1976 a 2001, compondo um banco de dados de 17.942 registros, oriundos de 2.582 animais, filhos de 186 touros e 1.035 matrizes. Foi utilizado um arquivo de pedigree contendo 6.353 animais, com 410 animais-base, sendo os pais dos animais nascidos a partir de 1976. Foram estudados os pesos obtidos desde o nascimento até o momento da seleção de machos, ou seja, final da PGP, quando os animais contavam com, aproximadamente, 13 meses de idade. Os registros de pesos foram coletados ao nascer e, em média, aos 120 dias, aos 210 dias, no início da PGP (220 dias), após o período de adaptação da PGP (260 dias), no período intermediário da PGP (320 dias) e no final da PGP (378 dias). Mesmo os animais sendo pesados em uma data fixa, caracterizando períodos discretos, as idades às pesagens apresentaram variação de aproximadamente três meses, em razão da estação de monta de 90 dias, fazendo com que as pesagens se distribuam em praticamente todas as idades possíveis.

Para consistência dos dados, foram considerados válidos os registros de animais que não apresentaram problemas crônicos de saúde, que apresentavam peso ao nascer superior a $15 \mathrm{~kg}$ e aqueles que na mesma classe de idade (60 dias) e grupo de contemporâneos (GC), definido como ano de nascimento e rebanho, tinham o peso dentro de uma amplitude de mais ou menos três desvios-padrão.

Foram extraídos do conjunto de dados original, além do arquivo de registros de pesos ao nascer, arquivos parcialmente sobrepostos, tomados em intervalos de idade de 60 dias e sobrepostos a cada 30 dias. Por exemplo, a idade 3 ( 3 meses em média) compreendeu o intervalo de 60 a 120 dias; a idade 4 , o intervalo de 90 a 150 dias; e assim sucessivamente até a idade 13, que compreendeu o intervalo de 360 a 420 dias. A sobreposição de idades possibilitou a obtenção de estimativas que podem ser consideradas contínuas. Cada arquivo continha somente um registro de peso por animal, com média de 2.261 animais e 2,28 filhos por matriz. A divisão dos arquivos em classes de 60 dias se deu em função do número restrito de animais em cada classe de idade se tomadas em intervalos menores. Essa distribuição dos registros cobre, de certa forma, toda a trajetória de crescimento até os 13 meses de idade.

Os componentes de variância foram encontrados para os pesos em cada classe de idade separadamente, usando-se o método da Máxima Verossimilhança Restrita - REML, que utiliza um algoritmo livre de derivadas, ajustado para um modelo animal, que incorporou todas as informações disponíveis de pedigree. As análises foram feitas por intermédio do programa computacional ASREML (Gilmour et al., 1999).

Os modelos de análise incluíram os efeitos fixos de grupos de contemporâneos, definidos por ano de nascimento (1978 a 2000) e rebanho (Original, Seleção, 
Controle e Tradicional), mês de nascimento $(7,8, \ldots, 11)$, idade da mãe em classes de anos $(3,4, \ldots, 11)$ e idade do animal na pesagem (dias) como covariável (efeito linear). Os efeitos fixos foram os mesmos para todos os modelos estudados, com exceção do peso ao nascer, que não incluiu a idade do animal.

Uma série de 36 análises univariadas com três modelos genéticos distintos foi desenvolvida. $\mathrm{O}$ modelo 1 (M1), ou modelo completo, considerou os efeitos aleatórios genéticos aditivos direto e materno, de ambiente permanente materno e residual; o modelo 2 (M2) ajustou para os efeitos genético aditivo direto, de ambiente permanente materno e residual; e o modelo 3 (M3) ajustou somente para os efeitos genético aditivo direto e residual.

Adicionalmente, a título de comparação, foram efetuadas análises univariadas de pesos padronizados para as médias de idades nas quais foram obtidos. Assim, uma segunda série de análises foi conduzida, considerando os pesos padronizados para 120 (P120), 210 (P210), 260 (P260), 320 (P320) e 378 (P378) dias de idade. Os modelos de análise foram os mesmos, com exceção da exclusão da idade na pesagem usada como covariável.

De acordo com análises preliminares, a inclusão da covariância entre os efeitos diretos e maternos $\left(\sigma_{\mathrm{am}}\right)$ diferente de zero nas análises do peso ao nascer, P120, P210 e P378 não mostrou diferenças significativas em relação ao modelo com $\sigma_{\mathrm{am}}$ igual a zero. Dessa forma, para o M1, assumiu-se covariância igual a zero entre os efeitos genéticos aditivos direto e materno.

Em notação matricial, o modelo completo utilizado pode ser descrito como:

$$
\mathrm{y}=\mathrm{Xb}+\mathrm{Z}_{1} \mathrm{a}+\mathrm{Z}_{2} \mathrm{~m}+\mathrm{Wc}+\mathrm{e}
$$

em que y é o vetor de observações, b é o vetor de efeitos fixos, a e m são os vetores de efeitos aleatórios genéticos aditivo direto e materno, $\mathbf{c}$ é o vetor de efeito de ambiente permanente materno, e é o vetor de efeitos residuais e $\mathbf{X}, \mathbf{Z}_{\mathbf{1}}, \mathbf{Z}_{\mathbf{2}}$ e $\mathbf{W}$ são as matrizes de incidência associadas a $\mathbf{b}, \mathbf{a}, \mathbf{m}$ e $\mathbf{c}$, respectivamente. $\mathrm{E}, \mathrm{E}(\mathrm{y})=\mathrm{Xb}, \mathrm{E}(\mathrm{a})=0, \mathrm{E}(\mathrm{m})=0 \mathrm{e}$ $\mathrm{E}(\mathrm{e})=0, \mathrm{e}$

$$
\mathrm{V}\left[\begin{array}{c}
\mathrm{a} \\
\mathrm{m} \\
\mathrm{c} \\
\mathrm{e}
\end{array}\right]=\left[\begin{array}{cccc}
A \sigma_{\mathrm{a}}^{2} & 0 & 0 & 0 \\
0 & A \sigma_{\mathrm{m}}^{2} & 0 & 0 \\
0 & 0 & I \sigma_{\mathrm{c}}^{2} & 0 \\
0 & 0 & 0 & I \sigma_{\mathrm{e}}^{2}
\end{array}\right]
$$

em que A é o numerador da matriz de parentesco entre os animais, I a matriz identidade e $\sigma_{a}^{2}, \sigma_{m}^{2}, \sigma_{c}^{2}$ e $\sigma_{\mathrm{e}}^{2}$ são as variâncias dos efeitos aleatórios $\mathbf{a}, \mathbf{m}, \mathbf{c}$ e e, respectivamente.

O teste de razão de verossimilhança (LRT) foi utilizado para verificar se os modelos diferem estatisticamente entre si, pela diferença entre os valores de $-2 \log \mathrm{L}$. Esse teste baseia-se na distribuição de qui-quadrado com $g$ graus de liberdade e probabilidade de erro de 5\%, em que $g$ é a diferença em números de parâmetros estimados nos modelos comparados (Dobson,1990).

\section{Resultados e Discussão}

A estrutura do banco de dados e as estatísticas descritivas das características são descritas na Tabela 1 . Houve considerável aumento nas médias dos pesos entre as classes subjacentes de idade, principalmente entre as classes 3 e 4 , em que a taxa de aumento foi de cerca de $20 \%$ em relação à média. Esse aumento, superior aos demais, se deve ao fato de o conjunto de dados conter, principalmente, animais gerados no último terço da estação de monta e, no momento da pesagem, que ocorre em média aos 120 dias de idade (P120), esses animais ainda serem muito jovens e com pesos inferiores aos demais. As médias de pesos variaram de 31,1 kg (PN) a 309,8 kg (384 dias de idade) e os desvios-padrão foram crescentes com a idade, evidenciando o efeito de escala oriundo do aumento das médias (Tabela 1). Os coeficientes de variação foram maiores no período pré-desmame (até sete meses), decrescendo até o final da PGP. Para os pesos padronizados (Tabela 1), houve tendência semelhante de aumento das médias, com maior crescimento na fase pré-desmame $(61 \%$ do peso total) e menor após esse período.

Foram detectadas diferenças significativas $(\mathrm{P}<0,05)$ pelo teste de razão de verossimilhança, para todas as idades quando os modelos completo (M1) e intermediário (M2) foram comparados com o modelo simples (M3) (Tabela 2). Com exceção do conjunto de pesos obtidos aos três meses de idade, que corresponde ao de menor número de dados e também o de menor proporção filhos/mãe (Tabela 1), os valores de $2 \log \mathrm{L}$ não diferiram significativamente $(\mathrm{P}<0,05)$ quando os modelos M1 e M2 (Tabela 2) foram comparados entre si, mostrando que, para esses conjuntos de dados, a decomposição do componente materno em genético e de ambiente

R. Bras. Zootec., v.33, n.6, p.1405-1415, 2004 
permanente não resultou em melhoria de ajuste. Pelicioni et al. (2003), em estudo de pesos mensais de animais Guzerá, obtidos do nascimento aos 450 dias de idade, da mesma forma, não encontraram diferenças significativas entre os modelos que consideraram os efeitos maternos em conjunto ou decompostos (genético e de ambiente permanente). Esses autores destacaram as dificuldades em decompor efeitos genéticos direto e materno e de ambiente materno em dados não experimentais. Entretanto, como pode ser observado no presente estudo, esse problema persiste também em dados experimentais e com estrutura de famílias bem definida.

Observa-se para a maioria dos conjuntos de dados estudados, que o modelo intermediário (M2), ajustado somente para efeito de ambiente permanente materno, apresentou a mesma eficácia de ajuste em relação ao modelo completo. Segundo Meyer (1992), a inclusão de um dos efeitos maternos no modelo de análise pode ser suficiente para ajustar-se para a variação decorrente de ambos os efeitos (genético materno e de ambiente permanente materno).

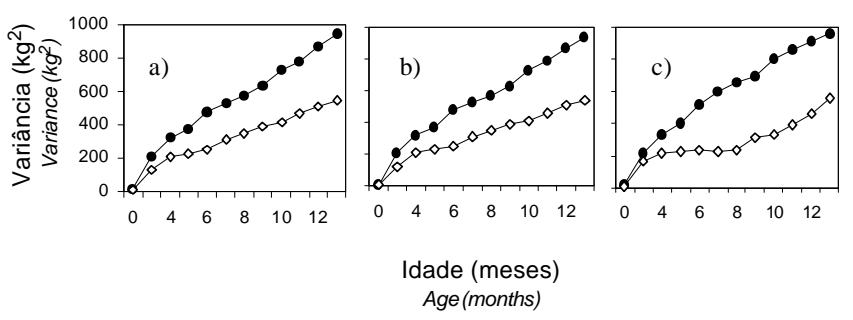

Figura 1 - Estimativas de variâncias fenotípicas $(\bullet)$ e residuais $(\diamond)$ para os modelos $M 1(a), M 2(b)$ e M3 (c), de acordo com a média de idade em meses.

Figure 1 - Estimates of phenotypic (๑), and residual $(\diamond)$ variances for the models M1 (a), M2 (b) and M3 (c), according to the average age in months.

Tabela 1 - Estrutura dos dados e estatísticas descritivas, para cada arquivo, dos pesos em idades subseqüentes e às idades padrão

Table 1 - Data structure and descriptive statistics, for each file, of the weights in subsequent ages and at the standardized ages

\begin{tabular}{|c|c|c|c|c|c|c|c|c|}
\hline $\begin{array}{c}\text { Característica }^{1} \\
\text { Trait }^{1}\end{array}$ & $\begin{array}{c}\text { Amplitude } \\
\text { Range }\end{array}$ & $\begin{array}{c}\text { Média de } \\
\text { idade (dias) } \\
\text { Means of } \\
\text { age (days) }\end{array}$ & $\begin{array}{l}\text { № de animais } \\
N \text {. of animals }\end{array}$ & $\begin{array}{l}\text { Peso (kg) } \\
\text { Weight }\end{array}$ & $\begin{array}{l}\mathrm{DP}(\mathrm{kg}) \\
S D(k g)\end{array}$ & $\begin{array}{l}\mathrm{CV}(\%) \\
C V(\%)\end{array}$ & $\begin{array}{l}\text { № de } \\
\text { mães } \\
N . \text { of } \\
\text { dams }\end{array}$ & $\begin{array}{c}\text { № de filhos/ } \\
\text { mãe } \\
\text { N. of progeny/ } \\
\text { dams }\end{array}$ \\
\hline
\end{tabular}

Pesos nas idades subseqüentes

Weights at subsequent ages

\begin{tabular}{ccccccccc}
\hline 0 & $(0)$ & 0 & 2582 & 31,1 & 4,18 & 13,43 & 1035 & 2,49 \\
3 & $(60-120)$ & 99 & 829 & 109,7 & 20,15 & 18,37 & 587 & 1,41 \\
4 & $(90-150)$ & 127 & 1881 & 131,9 & 24,50 & 18,37 & 931 & 2,02 \\
5 & $(120-180)$ & 150 & 2379 & 148,9 & 25,26 & 17,26 & 1005 & 2,38 \\
6 & $(150-210)$ & 183 & 2189 & 171,9 & 28,75 & 16,73 & 978 & 2,24 \\
7 & $(180-240)$ & 210 & 2534 & 187,5 & 28,34 & 15,11 & 1025 & 2,47 \\
8 & $(210-270)$ & 237 & 2552 & 202,5 & 29,34 & 14,49 & 1028 & 2,48 \\
9 & $(240-300)$ & 273 & 2513 & 224,7 & 31,35 & 13,95 & 1020 & 2,46 \\
10 & $(270-330)$ & 301 & 2570 & 246,1 & 34,46 & 14,00 & 1032 & 2,49 \\
11 & $(300-360)$ & 331 & 2573 & 268,8 & 35,23 & 13,11 & 1033 & 2,49 \\
12 & $(330-390)$ & 360 & 2385 & 290,4 & 37,91 & 13,05 & 998 & 2,39 \\
13 & $(360-420)$ & 384 & 1817 & 309,8 & 37,47 & 12,10 & 874 & 2,08 \\
\hline
\end{tabular}

Pesos às idades padrão

Standardized weights

\begin{tabular}{llllllll} 
P120 & 120 & 2479 & 125,9 & 19,76 & 15,69 & 1030 & 2,41 \\
P210 & 210 & 2477 & 197,7 & 27,35 & 13,83 & 1032 & 2,40 \\
P260 & 260 & 2464 & 224,7 & 33,31 & 14,82 & 1028 & 2,40 \\
P320 & 320 & 2477 & 277,0 & 37,09 & 13,37 & 1030 & 2,40 \\
P378 & 378 & 2476 & 318,0 & 40,15 & 12,62 & 1015 & 2,44 \\
\hline
\end{tabular}

1 0, 3, .., 13, P120, P210, P260, P320 e P378: pesos ao nascimento, aos três, ..., e aos 13 meses de idade, e pesos padronizados aos $120,210,260,320$ e 378 dias de idade, respectivamente.

2 DP: desvio-padrão. ${ }^{3} \mathrm{CV}$ : coeficiente de variação.

${ }^{1} 0,3, \ldots, 13, P 120, P 210$, P260, P320 e P378: birth weights, at three, ..., and at 13 months of age, and the standardized weights at $120,210,260,320$ and 378 days of age, respectively.

${ }^{2}$ SD: standard deviation. ${ }^{3}$ VC: variation coefficient.

\section{R. Bras. Zootec., v.33, n.6, p.1405-1415, 2004}


Na Figura 1 e na Tabela 3, são apresentadas as estimativas de variâncias fenotípicas $\left(\sigma_{p}^{2}\right)$ e residuais $\left(\sigma_{\mathrm{c}}^{2}\right)$ para os pesos em idades subsequientes para os três modelos. Observa-se, nos três modelos, menores valores de variância fenotípica no início do crescimento, em torno de $14,3 \mathrm{~kg}^{2}$, com aumento contínuo e "quase linear" com a idade, chegando a valores próximos de $940,0 \mathrm{~kg}^{2}$ no período final (13 meses). Embora a variância fenotípica seja responsável por toda a variação "visível" na população - e, por esse motivo, deva se apresentar com a mesma magnitude, independentemente do método e do modelo utilizados para decompor suas fontes aleatórias de variação -, valores distintos foram observados para esse parâmetro, ao longo do período estudado, entre os modelos 1 e 2 e o modelo 3, sendo que as estimativas de M3 (Figura 1c) foram ligeiramente maiores em relação aos demais modelos (Figuras 1a e 1b) com estimativas variando de $14,8 \mathrm{~kg}^{2}$ (nascimento) a $963,8 \mathrm{~kg}^{2}$ (13 meses).
$\mathrm{Na}$ Tabela 4, encontram-se as estimativas dos parâmetros genéticos e fenotípicos com respectivos erros-padrão para os pesos padronizados obtidos em análises univariadas, considerando-se os três modelos de análise. A mesma tendência crescente da observada nas análises de pesos nas idades subseqüentes foi confirmada em análises dos pesos nas idades padrão.

Estimativas de variâncias residuais $\left(\sigma_{\mathrm{e}}^{2}\right)$ altas foram encontradas para os três modelos, chegando a responder por $56 \%$ da variação total no final da PGP (13 meses) (Tabela 3). As variâncias residuais foram similares em toda a amplitude de idade para os modelos M1 e M2 (Figuras 1a e 1b; Tabela 3). Entretanto, conforme detectado pelo LRT, diferenças substanciais foram observadas entre estes modelos e o modelo M3 (Figura 1c e Tabelas 2 e 3). Maiores valores de $\sigma_{\mathrm{e}}^{2}$ em M3 eram esperados, uma vez que foi detectada a importância dos efeitos maternos nas fases de crescimento estudadas e portanto, no modelo

Tabela 2 - Valores de 2 Log L, de acordo com o modelo e arquivo estudados

Table 2 - Values of $2 \log L$, in agreement with the model and file studied

\begin{tabular}{|c|c|c|c|}
\hline $\begin{array}{c}\text { Característica } 1 \\
\text { Traits }^{1}\end{array}$ & $\begin{array}{l}\text { Modelo } 1^{2} \\
\text { Model } 1^{2}\end{array}$ & $\begin{array}{c}\text { Modelo } 2^{2} \\
\text { Model } 2^{2}\end{array}$ & $\begin{array}{c}\text { Modelo } 3^{2} \\
\text { Model } 3^{2}\end{array}$ \\
\hline \multicolumn{4}{|c|}{ Pesos nas idades subseqüentes } \\
\hline $\begin{array}{l}0 \\
3 \\
4 \\
5 \\
6 \\
7 \\
8 \\
9 \\
10 \\
11 \\
12 \\
13 \\
\end{array}$ & $\begin{array}{r}-9124,73^{\mathrm{A}} \\
-4952,83^{\mathrm{A}} \\
-12382,76^{\mathrm{A}} \\
-15996,08^{\mathrm{A}} \\
-15129,50^{\mathrm{A}} \\
-17878,04^{\mathrm{A}} \\
-18232,39^{\mathrm{A}} \\
-18218,74^{\mathrm{A}} \\
-18937,11^{\mathrm{A}} \\
-19193,29^{\mathrm{A}} \\
-18000,16^{\mathrm{A}} \\
-13779,21^{\mathrm{A}} \\
\end{array}$ & $\begin{array}{c}-9127,74^{\mathrm{A}} \\
-4959,27^{\mathrm{B}} \\
-12383,08^{\mathrm{A}} \\
-15996,63^{\mathrm{A}} \\
-15130,36^{\mathrm{A}} \\
-17878,87^{\mathrm{A}} \\
-18235,28^{\mathrm{A}} \\
-18221,15^{\mathrm{A}} \\
-18940,73^{\mathrm{A}} \\
-19194,80^{\mathrm{A}} \\
-18002,65^{\mathrm{A}} \\
-13780,67^{\mathrm{A}}\end{array}$ & $\begin{array}{c}-9152,17^{\mathrm{C}} \\
-4983,44^{\mathrm{C}} \\
-12451,29^{\mathrm{C}} \\
-16136,79^{\mathrm{C}} \\
-15285,52^{\mathrm{C}} \\
-18020,34^{\mathrm{C}} \\
-18357,63^{\mathrm{C}} \\
-18312,36^{\mathrm{C}} \\
-19021,10^{\mathrm{C}} \\
-19247,44^{\mathrm{C}} \\
-18039,66^{\mathrm{C}} \\
-13800,13^{\mathrm{C}}\end{array}$ \\
\hline \multicolumn{4}{|c|}{$\begin{array}{l}\text { Pesos às idades padrão } \\
\text { Standardized ages }\end{array}$} \\
\hline $\begin{array}{l}\text { P120 } \\
\text { P210 } \\
\text { P260 } \\
\text { P320 } \\
\text { P378 }\end{array}$ & $\begin{array}{l}-8122,20^{\mathrm{A}} \\
-8820,82^{\mathrm{A}} \\
-9187,73^{\mathrm{A}} \\
-9606,73^{\mathrm{A}} \\
-9451,74^{\mathrm{A}}\end{array}$ & $\begin{array}{l}-8124,46^{\mathrm{A}} \\
-8824,02^{\mathrm{A}} \\
-9188,51^{\mathrm{A}} \\
-9608,09^{\mathrm{A}} \\
-9452,19^{\mathrm{A}}\end{array}$ & $\begin{array}{l}-8224,18^{\mathrm{C}} \\
-8959,00^{\mathrm{C}} \\
-9269,71^{\mathrm{C}} \\
-9639,98^{\mathrm{C}} \\
-9472,61^{\mathrm{C}}\end{array}$ \\
\hline \multicolumn{4}{|c|}{ 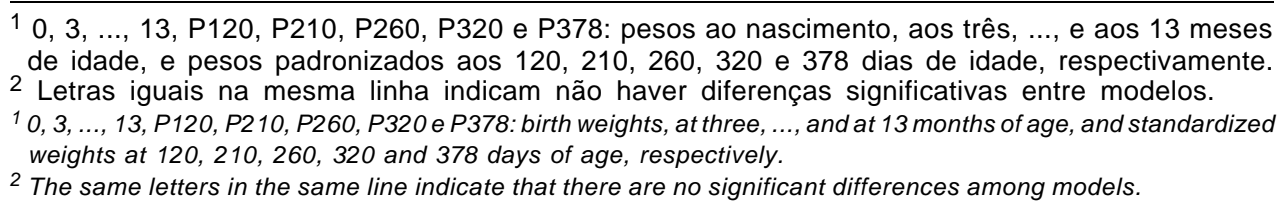 } \\
\hline
\end{tabular}


em que esses efeitos não foram considerados, alguma parte de sua variação deveria ser incluída na residual. No entanto, este parâmetro não apresentou essa tendência lógica em M3 e, em algumas idades (5 a 10 meses), foi inferior às registradas pelos modelos M1 e M2 (Tabela 3; Figuras 1a, 1b e 1c). Esses resultados sugerem que parte da variação anteriormente atribuída ao resíduo foi incorporada na variância genética direta, indicando mais um componente que contribuiu para a superestimação deste parâmetro. Nas análises de pesos padronizados (Tabela 4), a $\sigma_{\mathrm{e}}^{2}$ apresentou menor magnitude no M3 para todas as idades.
O problema da utilização de modelos que não consideram adequadamente os efeitos que contribuem efetivamente para a composição da variação fenotípica, no caso o M3, reside, principalmente, na redução da eficiência do processo de seleção pela obscuridade das relações entre genótipos e fenótipos (Lynch \& Walsh, 1997).

Na Figura 2 e na Tabela 3, são descritas as estimativas de variâncias genéticas aditiva direta $\left(\sigma_{\mathrm{a}}^{2}\right)$ e materna $\left(\sigma_{\mathrm{m}}^{2}\right)$ e de ambiente permanente materno $\left(\sigma_{\mathrm{c}}^{2}\right)$, para os pesos nas idades avaliadas e modelos utilizados. As estimativas de $\sigma_{a}^{2}$ foram

Tabela 3 - Estimativas de variâncias genéticas aditiva direta $\left(\sigma_{\mathrm{a}}^{2}\right)$ e materna $\left(\sigma_{\mathrm{m}}^{2}\right)$, de ambiente permanente materno $\left(\sigma_{c}^{2}\right)$ e residual $\left(\sigma_{e}^{2}\right)$, expressas em $\mathrm{kg}^{2}$, e herdabilidades direta( $\left.\mathrm{h}^{2}\right)$, materna $\left(\mathrm{m}^{2}\right)$ e variância de ambiente permanente materno como proporção da variância fenotípica $\left(c^{2}\right)$

Table 3 - Estimates of direct $\left(\sigma_{\mathrm{a}}^{2}\right)$ and maternal $\left(\sigma_{\mathrm{m}}^{2}\right)$ addictive genetic, maternal permanent environment $\left(\sigma_{\mathrm{c}}^{2}\right)$ and residual $\left(\sigma_{\mathrm{e}}^{2}\right)$ variances expressed in $\mathrm{kg}^{2}$, direct $\left(\mathrm{h}^{2}\right)$ and maternal $\left(\mathrm{m}^{2}\right)$ heritabilities and maternal permanent environmental effect as a proportion of the phenotypic variance $\left(c^{2}\right)$

\begin{tabular}{|c|c|c|c|c|c|c|c|c|c|c|c|c|}
\hline \multicolumn{13}{|c|}{$\begin{array}{l}\text { Pesos às idades subseqüentes (meses) } \\
\text { Weights at subsequent ages (months) }\end{array}$} \\
\hline & 0 & 3 & 4 & 5 & 6 & 7 & 8 & 9 & 10 & 11 & 12 & 13 \\
\hline \multicolumn{13}{|c|}{ Modelo 11} \\
\hline \multicolumn{13}{|c|}{ Model 11} \\
\hline$\sigma_{\mathrm{a}}^{2}$ & 5,29 & 11,28 & 26,08 & 29,16 & 39,02 & 46,17 & 49,10 & 78,04 & 128,96 & 168,58 & 200,49 & 248,24 \\
\hline$\sigma_{\mathrm{m}}^{2}$ & 0,72 & 44,07 & 6,75 & 9,09 & 16,54 & 18,64 & 33,38 & 33,45 & 45,47 & 29,58 & 44,67 & 44,53 \\
\hline$\sigma_{c}^{2}$ & 1,05 & 30,37 & 79,81 & 109,19 & 165,13 & 155,68 & 142,02 & 132,33 & 136,00 & 121,50 & 106,10 & 105,40 \\
\hline$\sigma_{8}^{2}$ & 7,20 & 126,11 & 209,92 & 227,19 & 255,56 & 311,33 & 350,82 & 391,46 & 416,68 & 466,18 & 514,61 & 545,69 \\
\hline$\sigma_{p}^{2}$ & 14,26 & 211,83 & 322,57 & 374,63 & 476,24 & 531,82 & 575,33 & 635,28 & 727,11 & 785,84 & 865,86 & 943,86 \\
\hline h2 & 0,37 & 0,05 & 0,08 & 0,08 & 0,08 & 0,09 & 0,09 & 0,12 & 0,18 & 0,21 & 0,23 & 0,26 \\
\hline e.p. & 0,06 & 0,07 & 0,04 & 0,03 & 0,04 & 0,04 & 0,04 & 0,04 & 0,05 & 0,05 & 0,06 & 0,07 \\
\hline $\mathrm{m}^{2}$ & 0,05 & 0,21 & 0,02 & 0,02 & 0,03 & 0,04 & 0,06 & 0,05 & 0,06 & 0,04 & 0,05 & $0,05 \mathrm{c} 2$ \\
\hline$c^{2}$ & 0,07 & 0,14 & 0,25 & 0,29 & 0,35 & 0,29 & 0,25 & 0,21 & 0,19 & 0,15 & 0,12 & 0,11 \\
\hline \multicolumn{13}{|c|}{$\begin{array}{l}\text { Modelo } 2 \\
\text { Model } 2\end{array}$} \\
\hline$\overline{\sigma_{a}^{2}}$ & 5,66 & 17,37 & 27,90 & 30,52 & 41,90 & 49,98 & 54,80 & 88,75 & 145,98 & 182,12 & 220,51 & 268,03 \\
\hline$\sigma_{c}^{2}$ & 1,56 & 70,70 & 85,76 & 117,56 & 179,38 & 171,70 & 170,48 & 159,46 & 171,57 & 144,15 & 139,69 & 137,54 \\
\hline$\sigma_{\mathrm{e}}^{\mathrm{c}}$ & 7,04 & 121,95 & 208,82 & 226,38 & 254,14 & 309,20 & 348,24 & 386,15 & 408,52 & 459,41 & 505,36 & 537,56 \\
\hline$\sigma_{p}^{2}$ & 14,26 & 210,02 & 322,49 & 374,46 & 475,41 & 531,18 & 573,52 & 634,36 & 726,07 & 785,68 & 865,57 & 943,13 \\
\hline$h^{2}$ & 0,40 & 0,08 & 0,09 & 0,08 & 0,09 & 0,09 & 0,09 & 0,14 & 0,18 & 0,23 & 0,25 & 0,28 \\
\hline e.p. & 0,06 & 0,07 & 0,04 & 0,04 & 0,04 & 0,04 & 0,04 & 0,04 & 0,05 & 0,05 & 0,06 & 0,07 \\
\hline$c^{2}$ & 0,11 & 0,34 & 0,27 & 0,31 & 0,38 & 0,32 & 0,30 & 0,25 & 0,24 & 0,18 & 0,16 & 0,15 \\
\hline \multicolumn{13}{|c|}{$\begin{array}{l}\text { Modelo } 3 \\
\text { Model } 3 \\
\end{array}$} \\
\hline$\overline{\sigma_{a}^{2}}$ & 8,23 & 43,70 & 119,56 & 175,78 & 275,48 & 372,14 & 422,00 & 385,31 & 470,42 & 470,00 & 452,55 & 408,36 \\
\hline$\sigma_{e}^{2}$ & 6,61 & 167,93 & 217,77 & 224,68 & 240,05 & 230,26 & 238,80 & 315,32 & 329,36 & 394,80 & 462,51 & 555,47 \\
\hline$\sigma_{p}^{2}$ & 14,85 & 211,62 & 337,34 & 400,47 & 515,53 & 602,40 & 661,71 & 700,62 & 799,78 & 864,80 & 915,07 & 963,83 \\
\hline$h^{2}$ & 0,55 & 0,21 & 0,35 & 0,44 & 0,53 & 0,62 & 0,64 & 0,55 & 0,59 & 0,54 & 0,49 & 0,42 \\
\hline e.p. & 0,05 & 0,09 & 0,07 & 0,06 & 0,06 & 0,05 & 0,05 & 0,06 & 0,05 & 0,06 & 0,06 & 0,07 \\
\hline
\end{tabular}

${ }_{1}^{1}$ e.p. $=$ erro-padrão da herdabilidade

${ }^{1}$ e.p. $=$ standard error of the heritabilities. 
similares para M1 e M2 ao longo do crescimento (Figuras 2a e 2b; Tabela 3), embora valores levemente inferiores tenham sido encontrados em M1 para peso ao nascer $\left(5,29 \mathrm{~kg}^{2}\right.$ vs. $\left.5,66 \mathrm{~kg}^{2}\right)$ e para peso ao final da PGP $\left(248,24 \mathrm{~kg}^{2}\right.$ vs. $\left.268,03 \mathrm{~kg}^{2}\right)$. Observaramse, para os modelos M1 e M2 (Tabela 3; Figuras 2a e $2 b$ ), valores de $\sigma_{a}^{2}$ ligeiramente crescentes até o desmame (7 meses) e súbito crescimento após esse período. Essa tendência foi verificada nos três modelos analisados, com maior intensidade no M3 (Figura 2c), em que o aumento de $\sigma_{a}^{2}$ apresentou-se acentuado desde o nascimento, provavelmente em razão da presença dos efeitos maternos nesse componente. Ainda no M3 (Figura 2c), houve ligeira queda de $\sigma_{\mathrm{a}}^{2}$ aos 9 meses e tendência quadrática dos 9 aos 13 meses de idade.

Tabela 4 - Estimativas de variâncias genéticas aditiva direta $\left(\sigma_{a}^{2}\right)$ e materna $\left(\sigma_{m}^{2}\right)$, de ambiente permanente materno $\left(\sigma_{\mathrm{c}}^{2}\right)$ e residual $\left(\sigma_{\mathrm{e}}^{2}\right)$, expressas em $\mathrm{kg}^{2}$, herdabilidades direta $\left(\mathrm{h}^{2}\right)$ e materna $\left(\mathrm{m}^{2}\right)$ e variấncia de ambiente permanente materno como proporção da variância fenotípica $\left(c^{2}\right)$

Table 4 - Estimates of direct $\left(\sigma_{a}^{2}\right)$ and maternal $\left(\sigma_{m}^{2}\right)$ addictive genetic, maternal permanent environment $\left(\sigma_{\mathrm{c}}^{2}\right)$ and residual $\left(\sigma_{\mathrm{e}}^{2}\right)$ variances expressed in $\mathrm{kg}^{2}$, direct $\left(h^{2}\right)$ and maternal $\left(\mathrm{m}^{2}\right)$ heritabilities and maternal permanent environmental effect as proportion of the total phenotypic variance $\left(c^{2}\right)$

\begin{tabular}{|c|c|c|c|c|c|}
\hline & \multicolumn{5}{|c|}{$\begin{array}{c}\text { Característica }^{1} \\
\text { Trait }\end{array}$} \\
\hline & P120 & P210 & P260 & P320 & P378 \\
\hline \multicolumn{6}{|c|}{ Modelo 1} \\
\hline \multicolumn{6}{|c|}{ Model 1} \\
\hline$\sigma_{a}^{2}$ & 10,72 & 33,44 & 75,38 & 144,48 & 273,98 \\
\hline$\sigma_{m}^{2}$ & 20,31 & 52,96 & 29,76 & 46,95 & 30,16 \\
\hline$\sigma_{c}^{2}$ & 77,24 & 175,92 & 194,80 & 131,98 & 130,90 \\
\hline$\sigma_{e}^{2}$ & 155,27 & 240,13 & 355,14 & 518,02 & 517,71 \\
\hline$\sigma_{p}^{2}$ & 263,54 & 502,45 & 655,08 & 841,43 & 952,75 \\
\hline $\mathrm{h}^{2}$ & $0,04 \pm 0,03$ & $0,07 \pm 0,03$ & $0,12 \pm 0,04$ & $0,17 \pm 0,05$ & $0,29 \pm 0,06$ \\
\hline $\mathrm{m}^{2}$ & $0,08 \pm 0,04$ & $0,11 \pm 0,05$ & $0,05 \pm 0,04$ & $0,06 \pm 0,04$ & $0,03 \pm 0,03$ \\
\hline $\mathrm{c}^{2}$ & $0,29 \pm 0,04$ & $0,35 \pm 0,05$ & $0,30 \pm 0,04$ & $0,16 \pm 0,04$ & $0,14 \pm 0,04$ \\
\hline \multirow{2}{*}{\multicolumn{6}{|c|}{$\begin{array}{l}\text { Modelo } 2 \\
\text { Model } 2\end{array}$}} \\
\hline & & & & & \\
\hline$\sigma_{\mathrm{a}}^{2}$ & 12,11 & 39,50 & 80,97 & 154,29 & 284,60 \\
\hline$\sigma_{\mathrm{c}}^{2}$ & 95,56 & 222,38 & 220,53 & 171,53 & 154,48 \\
\hline$\sigma_{\mathrm{e}}^{2}$ & 154,69 & 237,23 & 352,31 & 513,30 & 512,79 \\
\hline$\sigma_{p}^{2}$ & 262,36 & 499,11 & 653,81 & 839,12 & 951,87 \\
\hline $\mathrm{h}^{2}$ & $0,05 \pm 0,03$ & $0,08 \pm 0,03$ & $0,12 \pm 0,04$ & $0,18 \pm 0,05$ & $0,30 \pm 0,06$ \\
\hline $\mathrm{c}^{2}$ & $0,36 \pm 0,03$ & $0,45 \pm 0,03$ & $0,34 \pm 0,03$ & $0,20 \pm 0,03$ & $0,16 \pm 0,03$ \\
\hline \multicolumn{6}{|c|}{$\begin{array}{l}\text { Modelo } 3 \\
\text { Model } 3\end{array}$} \\
\hline$\sigma_{a}^{2}$ & 137,23 & 388,26 & 458,24 & 443,42 & 519,28 \\
\hline$\sigma_{\mathrm{e}}^{2}$ & 147,35 & 179,05 & 277,10 & 457,48 & 481,26 \\
\hline$\sigma_{p}^{2}$ & 284,58 & 567,31 & 735,34 & 900,90 & 1000,54 \\
\hline $\mathrm{h}^{2}$ & $0,48 \pm 0,06$ & $0,68 \pm 0,05$ & $0,62 \pm 0,05$ & $0,49 \pm 0,06$ & $0,52 \pm 0,05$ \\
\hline
\end{tabular}

\section{R. Bras. Zootec., v.33, n.6, p.1405-1415, 2004}


As estimativas das variâncias genéticas aditivas diretas estimadas neste estudo elevaram-se de forma irregular entre cada idade estudada. Foram encontradas taxas de aumento de cerca de $111,00 \%$ e $207,00 \%$ da primeira (nascimento) para a segunda idade ( 3 meses) para os modelos 1 e 2 , respectivamente. A partir dos quatro e até os sete meses de idade, os aumentos da $\sigma_{a}^{2}$ variaram de 11,80 a $33,00 \%$ e de 9,40 a $37,29 \%$ para M1 e M2, respectivamente. Entre sete e oito meses, idade que coincide com o período de adaptação da PGP, as taxas de aumento foram as menores em relação a todo o período estudado $(6,35$ e 9,64\% para M1 e M2, respectivamente). No início da PGP (pós-adaptação), as taxas de aumento das estimativas de $\sigma_{\mathrm{a}}^{2}$ apresentaram-se relativamente maiores, variando de 18,90 a $65,00 \%$ e de 21,70 a $64,50 \%$, para os modelos M1 e M2, respectivamente. Estimativas seguindo essa mesma tendência foram encontradas para os pesos padronizados (Tabela 4).

As estimativas das herdabilidades direta $\left(h^{2}\right)$ e materna $\left(\mathrm{m}^{2}\right)$ e das variâncias de ambiente permanente materno em relação à variância fenotípica total $\left(\mathrm{c}^{2}\right)$, para os modelos correspondentes, são apresentadas na Figura 3 e na Tabela 3, para os pesos nas idades subseqüentes e, na Tabela 4, para os pesos nas idades padronizadas. As herdabilidades diretas estimadas pelos modelos 1 e 2 foram essencialmente as mesmas (Figuras 3a e 3b), com valores ligeiramente maiores em algumas idades para M2. As pequenas diferenças observadas entre os modelos confirmam que o efeito

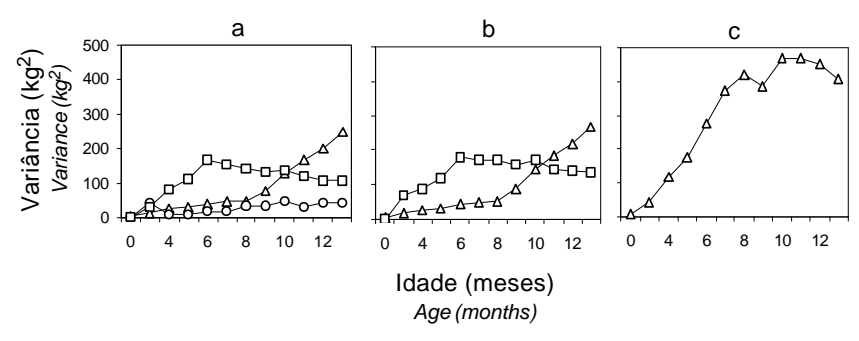

Figura 2 - Estimativas de variâncias genéticas aditivas direta $(\Delta)$ e materna $(\mathrm{O})$, de ambiente permanente materno ( $\square)$, para M1(a), M2(b) and M3 (c), de acordo com a média de idade, em meses.

Figure 2 - Estimates of addictive genetic direct $(\Delta)$ maternal $(\mathrm{O})$ and maternal permanent environment $(\boldsymbol{\square})$ variances for M1(a), M2(b) and M3 (c), according to the average age in months.

R. Bras. Zootec., v.33, n.6, p.1405-1415, 2004 de ambiente permanente materno provavelmente englobou os efeitos genéticos maternos sem, contudo, prejudicar as estimativas do componente genético direto. Com exceção do peso ao nascer $(0,40$ e 0,37$)$, valores baixos de herdabilidade direta $(0,05$ a 0,09 para M1 de 0,08 a 0,09 para M2) foram estimados para os pesos até os oito meses de idade (Figuras $3 \mathrm{a}$ e 3b) e, após esse período, foi observado aumento razoável, chegando ao máximo de 0,26 e 0,28 aos 13 meses de idade para M1 e M2, respectivamente. Em contraste, as estimativas de $\mathrm{h}^{2}$, obtidas pelo modelo 3 (Tabela 3 e Figura 3c), foram substancialmente maiores, com valor de 0,55 para o peso ao nascimento e variação de 0,21 a 0,64 ao longo das idades. Esses aumentos refletem a união das variâncias dos efeitos genéticos direto e materno e de ambiente permanente materno em uma única fonte de variação.

As estimativas de $\mathrm{h}^{2}$ dos pesos padronizados (Tabela 3) seguiram as mesmas tendências observadas nas estimativas encontradas para os pesos nas idades subsequientes, nas idades correspondentes, em todos os modelos. As estimativas de $\mathrm{h}^{2}$ registradas neste estudo pelos modelos M1 e M2 apresentaram-se, em grande parte da amplitude de idade estudada, dentro da variação genética conhecida para a população Nelore de Sertãozinho. Valores de $h^{2}$ de $0,41 \pm 0,04,0,10 \pm 0,03$ e $0,14 \pm 0,03$ para os pesos ao nascimento, aos 120 e aos 210 dias de idade, foram relatados por Trovo et al. (1996). As estimativas foram diferentes, principalmente, para o peso à

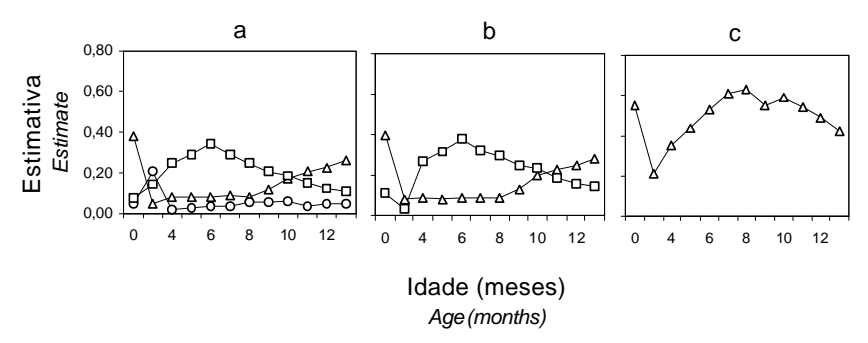

Figura 3 - Estimativas de herdabilidades direta $(\Delta)$ materna $(\mathrm{O})$ e do efeito de ambiente permanente materno como proporção da variância fenotípica (ם) para os modelos M1 (a), M2 (b) e M3 (c), de acordo com a média de idade, em meses.

Figure 3 - Estimates of direct ( $\Delta$ ) and maternal (O) heritabilities and maternal permanent environmental effect as proportion of phenotypic variances $(\boldsymbol{\square})$ for the models M1 (a), M2 (b) and M3 (c), by average of age in months. 
desmama ( 7 meses) das obtidas em revisões para populações zebuínas feitas por Mercadante et al. (1995) e Lôbo et al. (2000), que encontraram médias de estimativas de $h^{2}$ para os pesos ao nascer, ao desmame e aos 365 dias de idade, respectivamente, de 0,$33 ; 0,22$ e 0,20 e de 0,$29 ; 0,12$ e 0,22 . Estimativas de $h^{2}$ relatadas por Albuquerque \& Meyer (2001), com exceção do peso ao nascer, que foi bastante inferior $(0,28)$, foram similares às deste estudo para os modelos 1 e 2 , variando de 0,12 a 0,26 do período após o nascimento até 600 dias de idade e apresentando valores baixos $(0,12-0,13)$ nos períodos próximos ao desmame. Pelicioni et al. (2003), analisando pesos em idades subseqüentes de bovinos machos e fêmeas da raça Guzerá, também encontraram valores baixos para esse parâmetro, entretanto, com distribuição errática ao longo das idades, variando de 0,00 (420 dias) a 0,20 (450 dias de idade).

As estimativas de variâncias genética aditiva materna obtidas pelo modelo 1 (Figura 2a; Tabelas 3 e 4) foram de magnitude baixa, chegando ao máximo de 45,5 $\mathrm{kg}^{2}$ aos nove meses de idade, refletindo tendência crescente até os 13 meses nas idades subseqüentes (Figura 2a; Tabela 3), e ao máximo de $52,96 \mathrm{~kg}^{2}$ para o peso padronizado para 210 dias de idade (Tabela 4).

Os componentes de variância do efeito de ambiente permanente materno (Figuras 2a e 2b; Tabela 3) foram consideravelmente altos para os modelos 1 e 2, com aumento contínuo até os 6-7 meses de idade e diminuição gradual após esse período. As estimativas de $\sigma_{\mathrm{c}}^{2}$ superaram as de $\sigma_{\mathrm{a}}^{2}$ até os 10 meses de idade, quando a $\sigma_{a}^{2}$ passou a ser maior. Esses resultados diferem dos observados por Albuquerque \& Meyer (2001), que relataram efeitos de ambiente permanente materno de magnitude considerável, porém inferiores aos estimados para durante quase todo o crescimento, no período do nascimento aos 600 dias de idade, com exceção dos pesos obtidos aos 150 e aos 250 dias de idade, nos quais as duas estimativas foram muito próximas.

As estimativas de $c^{2}$ (Figuras 3a e 3b; Tabela 3) foram praticamente iguais para os modelos 1 e 2 , variando de 0,07 a 0,35 (M1) e de 0,06 a 0,38 (M2) para os pesos ao nascer e aos cinco meses de idade, respectivamente, com pequena diferença apenas aos três meses de idade, quando o valor foi inferior para o modelo 2, provavelmente em razão de problemas amostrais, pois trata-se do arquivo com menor número de animais e pequeno número de progênies por mãe
$(1,4)$ (Tabela 1). Nas Figuras 3a e 3b, fica claro que a contribuição do efeito de ambiente permanente materno para a variação fenotípica total é crescente até o desmame (7-8 meses), decrescendo após essa idade, mas mantendo-se como a mais importante até próximo dos 10 meses, quando, então, o efeito genético aditivo do próprio indivíduo passa a ser mais importante sem, no entanto, eliminar a importância do ambiente permanente materno. Essas tendências estão de acordo com os resultados obtidos para os pesos padronizados (Tabela 4). Albuquerque \& Meyer (2001) relataram variâncias de ambiente permanente materno como proporção da variância fenotípica total, que aumentaram do nascimento até os 150 dias de idade, permanecendo constante até 240 dias de idade e diminuindo gradualmente após esse período. Segundo Meyer (1993), efeitos maternos encontrados em pesos em idades avançadas ou pós-desmame são, possivelmente, conseqüência de efeitos em pesos anteriores. Neste estudo, isto pode ser visualizado na média do peso aos 13 meses (378 dias), que contém aproximadamente $62 \%$ do peso ao desmame (P210) (Tabela 1) e apresentou efeito de ambiente permanente materno de magnitude considerável.

\section{Conclusões}

A inclusão, no modelo de análise, do efeito de ambiente permanente materno foi suficiente para ajustar para a variação de ambos os efeitos maternos (genético e de ambiente permanente).

Os valores dos componentes de variâncias genéticas e ambientais são dependentes da idade do animal e apresentam mudanças importantes em curtos intervalos de tempo.

As estimativas de herdabilidade decresceram do nascimento aos três meses de idade, mantiveram-se baixas até o desmame e voltaram a aumentar após o desmame.

\section{Literatura Citada}

ALBUQUERQUE, L.G.; MEYER, K. Estimates of direct and maternal genetic effects for weights from birth to 600 days of age in Nelore cattle. Journal of Animal Breeding and Genetic, v.118, p.83-92, 2001.

CYRILLO, J.N.S.G.; RAZOOK, A.G.; FIGUEIREDO, L.A. et al. Efeitos da seleção para peso pós-desmame sobre medidas corporais e perímetro escrotal de machos Nelore de Sertãozinho (SP). Revista Brasileira de Zootecnia, v.29, n.2, p.444-453, 2000. 
DOBSON, A.J. An introduction to generalized linear models. Melbourne: Chapman and Hall, 1990. 174p.

GILMOUR, A.; CULLIS, B.R.; WELHAM, S.J. et al. ASREML Reference Manual. Orange: New South Wales Agriculture, 1999. 232p.

KIRKPATRICK, M. Selection on age-dependent traits. In: WORLD CONGRESS ON GENETICS APPLIED TO LIVESTOCK PRODUCTION., 7., 2002, Montpellier. Proceedings... Montpellier: [s.n.], 2002. CD-ROM.

KIRKPATRICK, M.; HILL, W.G.; THOMPSON, R. Estimating the covariance structure of traits during growth and ageing, illustrated with lactation in dairy cattle. Genetic Research, v.64, p.57-69, 1994.

KIRKPATRICK, M.; LOFSVOLD, D.; BULMER, M. Analysis of the inheritance, selection and evolution of growth trajectories. Genetics, v.124, p.979-993, 1990.

LYNCH, M.; WALSH, B. Genetics and analysis of quantitative traits. Sinauer Associates, 1997. 980p.

LÔBO, R.N.B.; MADALENA, F.E.; VIEIRA, A.R. Average estimates of genetic parameters for beef and dairy cattle in tropical regions. Animal Breeding Abstracts, v.68, p.433-462, 2000.

MERCADANTE, M.E.Z.; LÔBO, R.B.; REYES, A. de los. Parámetros genéticos para características de crecimiento en cebuínos de carne. Archivos Latinoamericanos de Produccion Animal, v.3, p.45-89, 1995.

MERCADANTE, M.E.Z.; PACKER, I.U.; RAZOOK, A.G. et al. Direct responses to selection for yearling weight on reproductive performance of Nelore cows. Journal of Animal Science, v.81, p.376-384, 2003

MEYER, K. Variance components due to direct and maternal effects for growth traits of Australian beef cattle. Livestock Production Science, v.31, p.142-144, 1992.

MEYER, K. Estimates of covariance components for growth traits of Australian Charolais cattle. Australian Journal Agricultural Research, v.44, p.1501-1508, 1993.

PELICIONI, L.C.; QUEIROZ, S.A.; ALBUQUERQUE, L.G. Estimativas de parâmetros genéticos para pesos ao nascer e mensais até 450 dias em bovinos Guzerá. Archivos Latinoamericanos de Produccion Animal, v.11, 2003. (prelo)

RAZOOK, A.G. Intensidades de seleção e mudanças genéticas em características de crescimento de rebanhos Nelore e Guzerá selecionados para peso pós-desmame. Relatório Anual do CNPq, 2002.
RAZOOK, A.G.; FIGUEIREDO, L.A.; BONILHA NETO, L.M. et al. O Programa de Seleção da Estação Experimental de Zootecnia de Sertãozinho: Resultados em 14 anos de progênies. In: SIMPÓSIO NACIONAL DE MELHORAMENTO ANIMAL, 1., 1996, Ribeirão Preto. Anais... Viçosa, MG: Sociedade Brasileira de Melhoramento Animal, 1996. p.204-208.

RAZOOK, A.G.; FIGUEIREDO, L.A.; BONILHA NETO, L.M. et al. Selection for yearling weight in Nelore and Guzerá zebu breeds: selection applied and response in 15 years of progeny. In: WORLD CONGRESS ON GENETICS APPLIED TO LIVESTOCK PRODUCTION, 6., 1998, Armidale. Proceedings... Armidale: University of New England, 1998. p.133-136.

RAZOOK, A.G.; FIGUEIREDO, L.A.; CYRILLO, J.N.S.G. et al. Prova de ganho de peso. Normas adotadas pela Estação Experimental de Zootecnia de Sertãozinho. Nova Odessa: Instituto de Zootecnia, 1997. 42p. (Boletim Técnico, 40).

RAZOOK, A.G.; FIGUEIREDO, L.A.; TROVO, J.B.F. et al. Intensidades de seleção e respostas direta e correlacionadas em 10 anos de progênies de bovinos das raças Nelore e Guzerá selecionadas para peso pós-desmame. Boletim Indústria Animal, v.50, n.2, p.147-163, 1993.

SAKAGUTI, E.S. Funções de covariâncias e modelos de regressão aleatória na avaliação genética do crescimento de bovinos jovens da raça Tabapuã. Viçosa, MG: Universidade Federal de Viçosa, 2000. 86p. Tese (Doutorado em Zootecnia) - Universidade Federal de Viçosa, 2000.

TROVO, J.B.F.; RAZOOK, A.G.; LÔBO, R.B. et al. Avaliações genéticas em bovinos de corte utilizando estruturas de dados não convencionais. In: CONGRESSO BRASILEIRO DE RAÇAS ZEBUÍNAS, 2., 1996, Uberaba. Anais... Uberaba: Associação Brasileira dos Criadores de Zebu, 1996. p.1-12.

Van Der WERF, J.H.J.; SCHAEFFER, L. Random regression in animal breeding. Guelph: CGIL Guelph, 1997. (Course Notes).

Recebido em: 07/06/03 Aceito em: 29/12/03 